\title{
2. On the uniform ergodic theorem concerning real linear operations.
}

\author{
By Shizuo KaKUTANI.
}

(Received February 15, 1940.)

1. Introduction. In the present paper, we shall prove that each proper value of modulus 1 of a real quasi-completely continuous linear operation $T$, whose iterates $T^{n}(n=1,2, \ldots)$ are uniformly bounded, is a root of unity. This is a generalization of a result of $\mathrm{K}$. Yosida [8]( ${ }^{1}$ ) in the operator-theoretical treatment of the Markoff process. Consequently, this may also be considered as a generalization of the results of M. Fréchet [3], J. L. Doob [2] and W. Doeblin [1] in the theory of concrete Markoff processes. We shall prove this theorem in an arbitrary Banach space. Since the proofs hitherto known are all restricted to the case of a concrete Markoff process, we have thus succeeded in taking a first step into the complete abstraction of the theory of Markoff processes.

In the last chapter of this paper, we shall prove that, in the special case of a matrix with non-negative elements, the condition of uniform boundedness may be omitted. This is a classical result of G. Frobenius [4]. But the proof given below, which appeals to the theory of Markoff processes, may be regarded with some interest.

2. The main theorem. Let $E$ be a real or a complex Banach space. A bounded linear operation $T$ which maps $E$ into itself is called to be completely continuous if $T$ maps the unit sphere of $E$ on a compact set of $E$. More generally, $T$ is called to be quasi-completely continuous if there exist an integer $m$ and a completely continuous linear operation $V$, which maps $E$ into itself, such that $\left\|T^{m}-V\right\|<1$.

We have previously investigated such a class of bounded linear operations in the operator-theoretical treatment of the Markoff process, and one of our principal results reads as follows:

Uniform ergodic theorem. Let $T$ be a quasi-completely continuous linear operation which maps a complex Banach space $E$ into itself. If there exists a constant $C$ such that $\left\|T^{n}\right\| \leqq C$ for $n=1,2, \ldots$, then the proper values of $T$ of modulus 1 are finite in number; and if we denote these by $\lambda_{1}, \lambda_{2}, \ldots, \lambda_{k}$, then $T^{n}$ is decomposed into the form:

(1) Numbers in brackets refer to the bibliography at the end of the paper. 


$$
T^{n}=\sum_{i=1}^{k}\left(\lambda_{i}\right)^{n} T_{\lambda_{i}}+S^{n}, \quad n=1,2, \ldots
$$

where $T_{i}(i=1,2 . \ldots, k)$ is a completely continuous linear operation which maps $E$ into itself such that $\left.\left.\left.\left.\left.\| T\rangle_{i} \| \leqq C,(T\rangle_{i}\right)^{2}=T\right\rangle_{i}, T T\right\rangle_{i}=T\right\rangle_{i} T=\lambda_{i} T\right\rangle_{i}$, $\left.\left.T \lambda_{i} T\right\rangle_{j}=0(i \neq j), T \lambda_{i} S=S T\right\rangle_{i}=0(i=1,2, \ldots, k)$ and $\left\|S^{n}\right\|<M \cdot(1+\varepsilon)^{-n}$ $(n=1,2, \ldots)$ with positive constants $M$ and $\varepsilon$.

The proof of this theorem is given in [5], [7] and [10].

Let now $E$ be a real Banach space and let $T$ be a bounded linear operation which maps $E$ into itself. In order to discuss the complex proper values of $T$, we shall introduce a Banach space $E^{*}=E+i E$ of all the points $z$ of the form: $z=x+i y, x \in E, y \in E$ with $\|z\|=\|x\|+\|y\|$ as its norm. If we put $\alpha z \equiv(a+i b)(x+i y)=(a x-b y)+i(b x+a y)$ $(\alpha=a+i b, a, b:$ real ; $x, y \in E)$, then $E^{*}$ is a complex Banach space and $T(z) \equiv T(x+i y)=T(x)+i T(y)$ is a bounded linear operatien which maps $E^{*}$ into itself. (It will be easily seen that we have $T(\alpha z)=\alpha T(z)$ for any complex number $\alpha$ and $z \in E$ ). Conversely, such a linear operation is called to be real. Strictly speaking, a point $z=x+i y$ is called to be real if we have $y=0$, and a bounded linear operation $T$ which maps $E$ into itself is called to be real if $z=$ real implies $T(z)=$ real.

The object of the present paper is to prove the

Theorem 1. Let $T$ be a quasi-completely continuous real linear operation which maps a complex Banach space $E^{*}=E+i E$ into itself. If there exists a constant $C$ such that $\left\|T^{n}\right\| \leqq C$ for $n=1,2, \ldots$, then all the proper values $\lambda$ of $T$ of modulus 1 , which are finite in number by the uniform ergodic theorem, satisfy the relation $\lambda^{N}=1$ with common $N$.

3. Proof of Theorem 1. By the uniform ergodic theorem, $T^{n}$ is decomposed into the form (1). We have only to prove that each proper value $\lambda_{i}$ of $T$ of modulus 1 is a root of unity, In order to show this, let us first introduce a notation $\sim$. Two complex numbers $\lambda$ and $\mu$ of modulus 1 are called to be equivalent, and this relation is denoted by $\lambda \sim \mu$, if there exists an integer $n$ such that $\lambda^{n}=\mu^{n}$. We shall prove that $\lambda_{i} \sim 1$ for $i=1,2, \ldots, k$. It is clear that we have

$$
\begin{aligned}
& \text { (i) } \lambda \sim \lambda, \\
& \text { (ii) } \lambda \sim \mu \text { implies } \mu \sim \lambda, \\
& \text { (iii) } \lambda \sim \mu \text { and } \mu \sim \nu \text { imply } \lambda \sim \nu .
\end{aligned}
$$

Hence the totality $\lambda_{1}, \lambda_{2}, \ldots, \lambda_{k}$ of all the proper values of $T$ of modulus 1 is decomposed into the classes $C_{\alpha}(\alpha=1,2, \ldots, l)$ of mutually equivalent numbers. Let us denote the elements of these classes $C_{\alpha}$ by 
$C_{\infty}=\left\{\lambda \alpha_{1}, \lambda \alpha_{2}, \ldots, \lambda \alpha_{d_{\alpha}}\right\}(\alpha=1,2, \ldots, l)$. For each $\alpha$, there exists an integer $n$ such that $\lambda_{\alpha_{1}}^{n}=\lambda_{\alpha_{2}}^{n}=\ldots=\lambda_{\alpha_{\alpha_{\alpha}}}^{n}$. Hence, if we take an integer $N$ sufficiently large, then we have $\lambda_{\alpha_{1}}^{N}=\lambda_{\alpha_{2}}^{N}=\cdots=\lambda_{\lambda_{d_{\alpha}}}^{N} \equiv \lambda_{\alpha}^{*}$ for $\alpha=1,2, \ldots, l$. It is clear that $\alpha \neq \beta$ implies $\lambda_{\alpha}^{*}+\lambda_{\beta}^{*}$ and that we may assume $\lambda_{\alpha}^{*}=1$ in case $\lambda_{\alpha}^{*} \sim 1$. Consequently (1) becomes

$$
T^{N n}=\sum_{\alpha=1}^{l}\left(\lambda_{\alpha}^{*}\right)^{n} T_{\alpha}^{*}+S^{N n}, \quad n=1,2, \ldots,
$$

where $\left.\left.T_{\alpha}^{*}=T\right\rangle_{\alpha_{1}}+T \lambda_{\alpha_{2}}+\cdots+T\right\rangle_{\alpha_{d}}$ and $\lambda_{\alpha}^{*}+\lambda_{\beta}^{*}$ for $\alpha \neq \beta$.

By the way, it is clear that we have $\left(T_{\alpha}^{*}\right)^{2}=T_{\alpha}^{*}, T^{N} T_{\alpha}^{*}=T_{\alpha}^{*} T^{N}=\lambda_{\alpha}^{*} T_{\alpha}^{*}$, $T_{\alpha}^{*} T_{\beta}^{*}=0(\alpha \neq \beta), T_{\alpha}^{*} S^{N}=S^{N} T_{\alpha}^{*}=0(\alpha=1,2, \ldots, l)$, i.e., (2) is the uniform ergodic decomposition of $T^{N}$ which corresponds to (1).

Since $\lambda_{\alpha}^{*}+\lambda_{\beta}^{*}$ for $\alpha \neq \beta$, there exists, by a theorem of Kronecker, for any system $\left\{\theta_{\alpha}\right\}(\alpha=1,2, \ldots, l)$ of complex numbers of modulus 1 (with $\theta_{\alpha}=1$ in case $\lambda_{\alpha}^{*}=1$ ), a sequence of positive integers $\left\{n_{\nu}\right\}$ $(\nu=1,2, \ldots)$ such that $\lim _{\nu \rightarrow \infty}\left(\lambda_{\alpha}^{*}\right)^{n_{\nu}}=\theta_{\alpha}$ for $\alpha=1,2, \ldots, l$. Hence, putting $n=n_{\nu}$ in (2) and making $\nu \rightarrow \infty$, we have (since $\left\|S^{n}\right\| \rightarrow 0$ as $n \rightarrow \infty$ )

$$
T^{N n} \nu \Rightarrow \sum_{\alpha=1}^{l} \theta_{\alpha} T_{\alpha}^{*} \quad \text { as } \quad \nu \rightarrow \infty,
$$

where $\Rightarrow$ means the convergence in the uniform topology of the operators (i.e., $\left\|T^{N n_{\nu}}-\sum_{\alpha=1}^{l} \theta_{\alpha} T_{\alpha}^{*}\right\| \rightarrow 0$ as $\left.\nu \rightarrow \infty\right)$. Since each $T^{N n_{\nu}}$ is a real operator, the right hand side of (3) must also be real. Hence, if there exists a $\lambda_{\alpha}^{*}$ with $\lambda_{\alpha}^{*}+1$, then, by putting $\theta_{\alpha}=$ an arbitrary complex number of modulus 1 and $\theta_{\beta}=1$ for $\beta \neq \alpha$, the bounded linear operation $\theta_{\alpha} T_{\alpha}^{*}+\sum_{\beta \neq \alpha} T_{\beta}^{*}$ must be real. Consequently, $\left(\theta_{\alpha}-\theta_{\alpha}^{\prime}\right) T_{\alpha}^{*} \equiv\left(\theta_{\alpha} T_{\alpha}^{*}+\sum_{\beta \neq \alpha} T_{\beta}^{*}\right)-\left(\theta_{\alpha}^{\prime} T_{\alpha}^{*}+\sum_{\beta \neq \alpha}^{* \neq \alpha} T_{\beta}^{*}\right)$ must be real for any complex numbers $\theta_{\alpha}$ and $\theta_{\alpha}^{\prime}$ with $\left|\theta_{\alpha}\right|=\left|\theta_{\alpha}^{\prime}\right|=1$. Hence $T_{\alpha}^{*}$ must vanish identically, and thus we have proved that $T_{\alpha}^{*}=0$ for any $\alpha$ with $\lambda_{\alpha}^{*}+1$. Consequently, each $\lambda_{i}$ belonging to the class $C_{\alpha}$ with $\lambda_{\alpha}^{*}+1$ satisfies

$$
T_{\lambda \alpha_{i}}=\sum_{j=1}^{d_{\alpha}} T_{\lambda \alpha_{i}} T_{\lambda \alpha_{j}}=T_{\lambda \alpha_{i}} \sum_{j=1}^{d_{\alpha}} T_{\lambda \alpha_{j}}=T_{\lambda \alpha_{i}} T_{\alpha}^{*}=0,
$$

i.e., $\lambda_{i}+1$ implies $T \lambda_{i}=0$. Thus we have proved that all $\lambda_{i}$ with $T_{i} \neq 0$ satisfies $\lambda_{i}^{N}=1$ for some integer $N$.

The proof of Theorem 1 is hereby completed.

4. Application of Theorem 1 to the theory of Markoff process. Let $P(t, E)$ be the transition probability that a point $t$ of an abstract 
space $\Omega$ is transferred, by a simple Markoff process, into a Borel set $E$ of $\Omega$ after the elapse of a unit-time. We have always $0 \leqq P(t, E) \leqq 1$ and $P(t, \Omega)=1$. We shall assume that $P(t, E)$ is completely additive in $E$ if $t$ is fixed and that $P(t, E)$ is Borel measurable in $t$ if $E$ is fixed. Then the transition probability $P^{(n)}(t, E)$ that a point $t \in \Omega$ is transferred into a Borel set $E$ of $\Omega$ after the elapse of $n$ unit-times is given recurrently by

$$
\begin{aligned}
P^{(n)}(t, E)=\int_{\Omega} P^{(n-1)}(t, d s) P(s, E) & \\
& n=2,3, \ldots ; P^{(1)}(t, E)=P(t, E),
\end{aligned}
$$

where the integration is of Radon-Stieltjes type.

The principal problem of the theory of Markoff process consists in the investigation of the behaviour of the sequence $\left\{P^{(n)}(t, E)\right\}(n=1,2, \ldots)$ for large $n$. The most appropriate method for this problem is perhaps that of the operator theory. We have often (see [6], [8], [10]) observed and made use of the fact that

$$
x \rightarrow T(x)=y: \quad y(E)=\int_{\Omega} x(d t) P(t, E)
$$

is a bounded positive linear operation of norm 1 which maps the Banach space $(M)$ of all the completely additive real valued set function $x(E)$ defined on the Borel sets $E$ of $\Omega$ (with $\|x\|=$ total variation of $x(E)$ on $\Omega$ as its norm) into itself, and that

$$
x \rightarrow T(x)=y: \quad y(t)=\int_{\Omega} P(t, d s) x(s)
$$

is a bounded positive linear operation of norm 1 which maps the Banach space $\left(M^{*}\right)$ of all the bounded Borel measurable real valued functions $x(t)$ defined on $\Omega$ (with $\|x\|=$ least upper bound of $|x(t)|$ on $\Omega$ as its norm) into itself. Thus the problem of the investigation of the asymptotic behaviour of the sequence $\left\{P^{(n)}(t, E)\right\}(n=1,2, \ldots)$ is transformed into that of the behaviour of the sequence of operators $\left\{T^{n}\right\}$ and $\left\{\bar{T}^{n}\right\}$ $(n=1,2, \ldots)$ for large $n$.

In this investigation, the principal rôle is played by the fact that, under certain conditions, all the proper values of $T$ and $\bar{T}$ of modulus 1 are roots of unity (see [7], [8], [10]). Although M. Fréchet, J. L. Doob and W. Doeblin did not appeal to the notions of operator theory, the same fact was essential in their proofs. M. Fréchet [3] discussed the case when $P(t, E)$ has a bounded density: $P(t, E)=\int_{E} p(t, s) m(d s)$, where 
$p(t, s)$ is a bounded measurable function defined on $\Omega \times \Omega$ and $m(E)$ is a completely additive measure given apriori on $\Omega($ with $m(\Omega)=1)$; J. L. Doob [2] required that there exists for any $\varepsilon>0$ an $\eta>0$ such that $m(E)<\eta$ implies $P(t, E)<\varepsilon$ for any $t \in \Omega$; and lastly, W. Deoblin [1] assumed that there exists two constants $\eta>0$ and $b>0$ such that $m(E)<\eta$ implies $P(t, E)<1-b$ for any $t \in \Omega$. It is, however, proved by K. Yosida [8] (see also [10]) that all these assumptions imply the quasi-complete continuity of $T$ and $\bar{T}$, and under this last condition we have investigated the asymptotic behaviour of the operations $T$ and $\bar{T}$ for large $n$ (see [9], [10]).

In our previous investigations, we first proved that the uniform ergodic theorem holds for such a class of linear operations, and then, using this uniform ergodic theorem, we have proved that all the proper values of $T$ of modulus 1 are roots of unity. But the fact that $T$ and $\bar{T}$ are the operators in the Banach spaces $(M)$ and $\left(M^{*}\right)$ respectively was essential in proof, while the proof given above is completely free from these restrictions. Thus a complete abstract treatment of the Markoff process becomes possible by Theorem 1 . It is, however, important to be noted that we have made use only of the fact that $T$ and $\bar{T}$ are real, and that the positiveness of these operators was not necessary in our arguments. An abstract theory of Markoff processes, where the positiveness of the operations $T$ and $\bar{T}$ plays its essential rôle, will be discussed on another occasion.

5. Matrix with non-negative elements. Let us now consider the case when $E$ is of finite dimension. In this case, Theorem 1 is reduced to

Theorem 2. Let $P=\left(p_{i j}\right)(i, j=1,2, \ldots, r)$ be a matrix with real elements, and let $P^{n}=\left(p_{i j}^{(n)}\right)(i, j=1,2, \ldots)$ be its $n$-th iterate. If there exists a constant $C$ such that $\left\|P^{n}\right\| \leqq C$ for $n=1,2, \ldots$, then all the proper values of $P$ of modulus 1 are roots of unity.

Remark. A finite dimensional Banach space $E$ can be equipped with many different norms which are mutually equivalent. In all these cases, the uniform boundedness of $\left\{\left\|P^{n}\right\|\right\}(n=1,2, \ldots)$ is equivalent to the uniform boundedness of $\left\{\left|p_{i j}^{(n)}\right|\right\}(i, j=1,2, \ldots, r ; n=1,2, \ldots)$.

In the following lines, we shall show that if all the elements $p_{i j}$ $(i, j=1,2, \ldots, r)$ are non-negative, then the condition of uniform boundedness of $\left\{\left\|p^{n}\right\|\right\}(n=1,2, \ldots)$ may be omitted. Indeed, we shall prove

Theorem 3. Let $P=\left(p_{i j}\right)(i, j=1,2, \ldots, r)$ be a matrix with real non-negative elements. If the maximum modulus of all the proper values of $P$ is 1 , then 1 is a proper ualue of $P$ and all proper values of $P$ of modulus 1 are roots of unity. 
Proof. $\left({ }^{2}\right)$ We shall first show that 1 is a proper value of $P$ and that there exists a positive proper element $x=\left(x_{1}, x_{2}, \ldots, x_{r}\right)\left(x_{i} \geqq 0\right.$, $i=1,2, \ldots, r$ and $x_{i}>0$ at least for one $i$ ) of $P$ belonging to the proper value 1. Let $y=\left(y_{1}, y_{2}, \ldots, y_{r}\right)$ be a proper element of $P$ belonging to the proper value $\lambda$ with $|\lambda|=1: \sum_{j=1}^{r} p_{i j} y_{j}=\lambda y_{i}, i=1,2, \ldots, r$. Then we have $\sum_{j=1}^{r} p_{i j}\left|y_{j}\right| \geqq\left|y_{i}\right|$ for $i=1,2, \ldots, r$. Hence there exists, by a result $\left({ }^{3}\right)$ concerning general completely continuous positive linear operations in semi-ordered Banach spaces, a positive value $\lambda_{0} \geqq 1$ and a positive proper element $x=\left(x_{1}, x_{2}, \ldots, x_{r}\right)\left(x_{i} \geqq 0, i=1,2, \ldots, r\right.$ and $x_{i}>0$ at least for one $i$ ) belonging to the proper value $\lambda_{0}: \sum_{j=1}^{r} p_{i j} x_{j}=\lambda_{0} x_{i}$, $i=1,2, \ldots, r$. Since the maximum modulus of proper values of $P$ is 1 by assumption, we must have $\lambda_{0}=1$ and our proposition is proved.

We shall divide our further discussions into two cases:

1st case: $x_{i}>0$ for any $i$. Put $p_{i j}^{*}=x_{i}^{-1} p_{i j} x_{j}$ for $i, j=1,2, \ldots, r$. Then $P^{*}=\left(p_{i j}^{*}\right)(i, j=1,2, \ldots, r)$ is a matrix with non-negative elements, and, since $\sum_{j=1}^{r} p_{i j}^{*}=x_{i}^{-1} \sum_{j=1}^{r} p_{i j} x_{j}=x_{1}^{-1} x_{i}=1$ for $i=1,2, \ldots, r, P^{*}$ defines a Markoff process with $\stackrel{j=1}{r}$ possible states. Hence $\left\{\left\|\left(P^{*}\right)^{n}\right\|\right\}(n=1,2, \ldots)$ is uniformly bounded. Consequently, by Theorem 2, all the proper values of $P^{*}$ of modulus 1 are roots of unity. Since clearly $P^{*}$ and $P$ have all their proper values in common, the proof of Theorem 2 is completed in the 1st case.

2nd case: $x_{i}=0$ for some $i$. We may assume that there exists an integer $s$ with $1 \leqq s<r$ such that $x_{i}>0$ for $1 \leqq i \leqq s$ and $x_{i}=0$ for $s<i \leqq r$. Since $\sum_{j=1}^{r} p_{i j} x_{j}=x_{i}$ for $i=1,2, \ldots, r$, we must have $p_{i j}=0$ for $i>s$ and $j \leqq s$. Consequently, $P$ is decomposed into the form:

$$
P=\left(\begin{array}{cc}
P_{1} & * \\
0 & P_{2}
\end{array}\right)
$$

where $P_{1}$ and $P_{2}$ are square matrices of degree $s$ and $r-s$ respectively. Since the totality of all the proper values of $P$ coincides with the totality

(2) The author expresses his hearty thanks to S. Iyanaga, K. Kodaira and M. Abe for their kind discussions concerning the proof of this theorem.

(3) Theorem. Let $T$ be a completely continuous positive linear operation which maps a semi-ordered Banach space into itself. If there exist a positive number $\mu>0$ and a positive element $y>0$ such that $T(y) \geqq \mu y$, then there exist a positive proper value $\lambda(\geqq \mu>0)$ of $T$ and a positive proper element $x>0$ of $T$ belonging to the proper value $\lambda: T(x)=\lambda \cdot x$.

This result was obtained by A. Rutmann: Sur une classe spécielle d'opérateurs linéaires totalement continues, C. R. URSS, $18(1938)$, No. 9. The proof of A. Rutmann relies on Schauder's fixed-point theorem. If $E$ satisfies the condition : $x \geqq y \geqq 0$ implies $\|x\| \geqq\|y\|$, then this result may be obtained directly by an elementary way (without appealing to the fixed-point theorem). The proof will be given in another paper. 
of all the proper values of $P_{1}$ and $P_{2}$, our theorem is thus reduced to the lower dimensional case. Since our theorem is clearly true for a one-dimensional case, the proof of Theorem 3 is completed by mathematical induction.

Remark. In proving Theorem 3, we have made use of one result, whose proof we have left to another paper. In order to avoid this difficulty, we may proceed as follows:

First, we may assume that $\sum_{i=1}^{r} p_{i j}>0$ for $j=1,2, \ldots, r$. For, if there exists a $j$ such that $\sum_{i=1}^{r} p_{i j}=0$, i.e. $p_{i j}=0$ for $i=1,2, \ldots, r$, then, by interchanging simultaneously the rows and columns of $P$, we arrive at a form (7) with $s=1$ and $P_{1} \equiv 0$. Hence our problem is reduced to the lower dimensional case.

Next, it will be easily seen that a matrix $P=\left(p_{i j}\right)(i, j=1,2, \ldots, r)$ with non-negative elements such that $\sum_{i=1}^{t} p_{i j} \geqq \delta>0$ for $j=1,2, \ldots, r$, has at least one positive proper value $\lambda_{0}$ and a positive proper element $x=\left(x_{1}, x_{2}, \ldots, x_{r}\right)\left(x_{i} \geqq 0, i=1,2, \ldots, r\right.$ and $x_{i}>0$ at least for one $\left.i\right)$ belonging to the proper value $\lambda_{0}: \sum_{j=1}^{r} p_{i j} x_{j}=\lambda_{0} x_{i}, i=1,2, \ldots, r$. Indeed, if we consider the totality $\Delta$ of all $y=\left(y_{1}, y_{2}, \ldots, y_{r}\right)$ with $\sum_{i=1}^{r} y_{i}=1$ and $y_{i} \geqq 0(i=1,2, \ldots, r)$, then $y \rightarrow \varphi(y)=z$, where $z=\left(z_{1}, z_{2}, \ldots, z_{r}\right)$, $z_{i}=\left(\sum_{i=1}^{r} \sum_{j=1}^{r} p_{i j} y_{j}\right)^{-1} \sum_{j=1}^{r} p_{i j} y_{j}(i=1,2 \ldots, r)$, is a continuous mapping of $\Delta$ into itself. (Note that $\sum_{i=1}^{r} \sum_{j=1}^{r} p_{i j} y_{j} \geqq \delta>0$ for any $y \in \Delta$ ). Since $\Delta$ is closed and convex, there exists a fixed-point $x \in \Delta$ with $\phi(x)=x$; i.e., $x=\left(x_{1}, x_{2} \ldots, x_{r}\right)$ satisfies $x_{i}=\left(\sum_{i=1}^{r} \sum_{j=1}^{r} p_{i j} x_{j}\right)^{-1} \sum_{j=1}^{r} p_{i j} x_{j}$ for $i=1,2, \ldots, r$. If we put $\lambda_{0}=\sum_{i=1}^{r} \sum_{j=1}^{r} p_{i j} x_{j} \geqq \delta>0$, then these $\lambda_{0}$ and $x=\left(x_{1}, x_{2}, \ldots, x_{r}\right)$ are the required ones.

Thus we have proved that there exist a positive proper value $\lambda_{0}>0$ and a positive proper element $x=\left(x_{1}, x_{2}, \ldots, x_{r}\right)$ belonging to the proper value $\lambda_{0}$. By assumption, we must have $\lambda_{0} \leqq 1$. Again our further discussions are divided into two cases:

1st case: $x_{i}>0$ for any $i$. In this case, $p_{i j}^{*}=x_{i}^{-1} p_{i j} x_{j}(i, j=1,2, \ldots, r)$ satisfy $\sum_{j=1}^{r} p_{i j}^{*}=\lambda_{0}$ for $i=1,2 \ldots, r$. If we have $\lambda_{0}=1$, then our problem is reduced to the case of a Markoff process, while in case $\lambda_{0}<1$ all the proper values of a matrix $p^{*}=\left(p_{i j}^{*}\right)(i, j=1,2, \ldots, r)$ and consequently those of $P=\left(p_{i j}\right)(i, j=1,2, \ldots, r)$ do not exceed $\lambda_{0}<1$ in absolute value, which is clearly a contradiction to the assumption that the maximum modulus of all the proper values of $P$ is 1 . 
On the uniform ergodic theorem concerning real linear operations.

2nd case: $x_{i}=0$ for some $i$. In this case, $P$ is decomposed into the form (7), and our problem is again reduced to the case of a lower dimensional matrix.

\section{Bibliography.}

(1) W. Doeblin: Sur les propriétés asymptotiques de mouvements régis par certains types de chaines simples, Bull. Math. Soc. Roumaine des Sci., 39-2 (1937), 3-61.

(2) J.L. Doob: Stochastic process with an integral valued parameter, Trans. Amer. Math. Soc., 44 (1938), 87-150.

(3) M. Fréchet: Sur l'allure asymptotique des densités itérés dans le problème des probabilités en chaîne, Bull. Soc. Math. France, 62 (1934), 68-83.

(4) G. Frobenius: Über Matrizen aus nicht negativen Elementen, Sitzungsberichte der phys-math. Klasse, Akademie Berlin, 1912, 456-477.

(5) S. Kakutani: Iteration of linear operations in complex Banach spaces, Proc. Imp. Acad. Japan, 14 (1938), 295-300.

(6) S. Kakutani : Some results in the operator-theoretical treatment of the Markoff process, Proc. Imp. Acad. Japan, 15 (1939), 260-264.

(7) K. Yosida: Abstract integral equations and the homogeneous stochastic processes, Proc. Imp. Acad. Japan, 14 (1938), 287-291.

(8) K. Yosida: Operator-theoretical treatment of the Markoff process, Proc. Imp. Acad. Japan, 14 (1938), 363-367.

(9) K. Yosida: Operator-theoretical treatment of the Markoff process, II, Proc. Imp. Acad. Japan, 15 (1939), 127-130.

(10) K. Yosida and S. Kakutani : Operator-theoretical treatment of mean ergodic theorem and Markoff process, forthcoming in Annals of Mathematics.

\section{Mathematical Institute, Osaka Imperial University.}

